\title{
On the Global Dynamics of a Vector-Borne Disease Model with Age of Vaccination
}

\author{
Stanislas Ouaro $\mathbb{D}$ and Ali Traoré \\ LAboratoire de Mathématiques et Informatique (LAMI), Unité de Formation et de Recherche en Sciences Exactes et Appliquées, \\ Département de Mathématiques, Université Ouaga I Pr Joseph KI-ZERBO, BP 7021, Ouagadougou, Burkina Faso
}

Correspondence should be addressed to Ali Traoré; traoreali.univ@yahoo.fr

Received 22 August 2017; Revised 11 January 2018; Accepted 8 February 2018; Published 15 March 2018

Academic Editor: Khalid Hattaf

Copyright (C) 2018 Stanislas Ouaro and Ali Traoré. This is an open access article distributed under the Creative Commons Attribution License, which permits unrestricted use, distribution, and reproduction in any medium, provided the original work is properly cited.

\begin{abstract}
We study a vector-borne disease with age of vaccination. A nonlinear incidence rate including mass action and saturating incidence as special cases is considered. The global dynamics of the equilibria are investigated and we show that if the basic reproduction number is less than 1, then the disease-free equilibrium is globally asymptotically stable; that is, the disease dies out, while if the basic reproduction number is larger than 1 , then the endemic equilibrium is globally asymptotically stable, which means that the disease persists in the population. Using the basic reproduction number, we derive a vaccination coverage rate that is required for disease control and elimination.
\end{abstract}

\section{Introduction}

Many of infections that have the important impact on human health in terms of mortality or morbidity are vector-borne disease. Mosquitoes [1] are perhaps the best known disease vectors, with various species playing a role in the transmission of infections such as malaria, yellow fever, dengue fever, and West Nile virus. One of the effective methods in disease prevention is the vaccination [2-5]. Several studies in the literature have been carried out to investigate the role of treatment and vaccination of the spread of diseases ([6-8] and the references therein). An epidemic model with vaccination for measles is derived by Linda [9]. The effect of vaccination on the spread of periodic diseases, using discrete-time model, was studied by Mickens [10].

The impact of vaccination in two SVIR models with permanent immunity is studied by Liu et al. [11]. Xiao and Tang [12] have shown from an SIV model that complex dynamics are induced by imperfect vaccination. Gumel and Moghadas [13] investigated a disease transmission model by considering the impact of a protective vaccine and found the optimal vaccine coverage threshold required for disease control and elimination. The eradicating of an SEIRS epidemic model by using vaccine was studied by Gao et al. [14]. Yang et al. [8] derived a threshold value for the vaccination coverage of an SIVS epidemic model. Many previous studies have shown that the reemergence of some diseases is caused by the waning of vaccine-induced immunity [15-17]. A consequence of this is that it is important for health authorities to take into account waning of vaccine-induced immunity in the disease control and elimination campaign.

In this paper, we consider a vector-borne disease model such as malaria that incorporates the waning of vaccineinduced immunity. Additionally, we use incidences with a nonlinear response to the number of infectious individuals and infectious vectors. The incidences take the form $S f(I)$ and $S g(I)$, respectively, for the human and vector populations. We assume that $f$ and $g$ satisfy the following assumptions:

(H1) For $x \in \mathbb{R}^{+}, f(x) \geq 0$ with equality if and only if $x=0, f^{\prime}(x) \geq 0$, and $f^{\prime \prime}(x) \leq 0$.

(H2) For $x \in \mathbb{R}^{+}, g(x) \geq 0$ with equality if and only if $x=0, g^{\prime}(x) \geq 0$, and $g^{\prime \prime}(x) \leq 0$.

From the above assumptions and the Mean Value Theorem, it follows that

$$
\begin{aligned}
& f^{\prime}(x) x \leq f(x) \leq f^{\prime}(0) x, \\
& g^{\prime}(x) x \leq g(x) \leq g^{\prime}(0) x .
\end{aligned}
$$


Let $S_{h}, I_{h}$, and $R_{h}$ denote, respectively, the number of susceptible, infectious, and removed host individuals and $S_{v}, I_{v}$ the number of susceptible and infectious vectors. The susceptible individuals are vaccinated at the rate $\theta \geq 0 . v(t, a)$ denotes the population size of the vaccinated compartment at time $t$ with the vaccine age $a$. Let $\epsilon(a)$ be the rate at which the vaccine-induced immunity wanes. We assume that $\epsilon(a)$ and the following assumption:

$(\mathbf{H 3}) \epsilon:[0, \infty) \rightarrow[0, \infty)$ is bounded, nondecreasing, and piecewise continuous with possibly many finite jumps.

We consider a relatively isolated community where there is no immigration or emigration. Additionally, we assume that all the newly recruited, including the newborns, are susceptibles. Let, at any time $t, \Lambda_{h}$ and $\Lambda_{v}$ be the recruitment rate of host individuals and vectors, respectively. $\mu_{h}$ and $\mu_{v}$ are, respectively, the natural death rate of host individuals and vectors. Let $\gamma$ be the natural recovery rate from the infected population and $\delta$ the disease induced death rate of host individuals. The number of individuals moving from the vaccinated class into the susceptible class at time $t$ is $\int_{0}^{+\infty} v(t, a) d a$. From the above assumptions, we formulate our vector-borne epidemic model in the following way:

$$
\begin{aligned}
\frac{d S_{h}(t)}{d t}= & \Lambda_{h}-\mu_{h} S_{h}(t)-S_{h}(t) f\left(I_{v}(t)\right) \\
& -\theta S_{h}(t)+\int_{0}^{\infty} \epsilon(a) v(t, a) d a, \\
\frac{d I_{h}(t)}{d t} & =S_{h}(t) f\left(I_{v}(t)\right) \\
\frac{\partial v(t, a)}{\partial t}+\frac{\partial v(t, a)}{\partial a} & =-\left(\mu_{h}+\gamma+\delta\right) I_{h}(t), \\
\frac{d R_{h}(t)}{d t} & =\gamma I_{h}(t)-\mu_{h} R_{h}(t), \\
\frac{d S_{v}(t)}{d t} & =\Lambda_{v}-\mu_{v} S_{v}(t)-S_{v}(t) g\left(I_{h}(t)\right), \\
\frac{d I_{v}(t)}{d t} & =S_{v}(t) g\left(I_{h}(t)\right)-\mu_{v} I_{v}(t), \\
v(t, 0) & =\theta S_{h}(t), \\
S_{h}(0) & =S_{h 0} \geq 0, \\
I_{h}(0) & =I_{h 0}>0, \\
R_{h}(0) & =R_{h 0} \geq 0, \\
v(0, \cdot) & =v_{0}(\cdot) \in L_{+}^{1}, \\
S_{v}(0) & =S_{v 0} \geq 0, \\
I_{v}(0) & =I_{v 0}>0, \\
&
\end{aligned}
$$

where $L_{+}^{1}$ is the set of integrable functions from $(0, \infty)$ into $\mathbb{R}^{+}=[0, \infty)$. Since the removed host individual population does not appear in the remaining equations of system (2), it is sufficient to consider the following system:

$$
\begin{aligned}
& \frac{d S_{h}(t)}{d t}=\Lambda_{h}-\mu_{h} S_{h}(t)-S_{h}(t) f\left(I_{v}(t)\right) \\
& -\theta S_{h}(t)+\int_{0}^{\infty} \epsilon(a) v(t, a) d a, \\
& \frac{d I_{h}(t)}{d t}=S_{h}(t) f\left(I_{v}(t)\right) \\
& -\left(\mu_{h}+\gamma+\delta\right) I_{h}(t), \\
& \frac{\partial v(t, a)}{\partial t}+\frac{\partial v(t, a)}{\partial a}=-\left(\mu_{h}+\epsilon(a)\right) v(t, a), \\
& \frac{d S_{v}(t)}{d t}=\Lambda_{v}-\mu_{v} S_{v}(t)-S_{v}(t) g\left(I_{h}(t)\right), \\
& \frac{d I_{v}(t)}{d t}=S_{v}(t) g\left(I_{h}(t)\right)-\mu_{v} I_{v}(t), \\
& v(t, 0)=\theta S_{h}(t), \\
& S_{h}(0)=S_{h 0} \geq 0, \\
& I_{h}(0)=I_{h 0}>0 \text {, } \\
& R_{h}(0)=R_{h 0} \geq 0 \text {, } \\
& v(0, \cdot)=v_{0}(\cdot) \in L_{+}^{1}, \\
& S_{v}(0)=S_{v 0} \geq 0 \text {, } \\
& I_{v}(0)=I_{v 0}>0 \text {. }
\end{aligned}
$$

From $[18,19]$, we state that system (3) has a unique continuous solution if the initial conditions satisfy the compatibility condition

$$
v_{0}(0)=\theta S_{h 0}
$$

In the remaining part of this paper, we always assume that condition (4) is satisfied. The existence and the nonnegativity of the solution of (3) can be reached in Browne and Pilyugin [20]. We next introduce a semiflow solution of system (3).

Define

$$
\begin{aligned}
\chi & =\mathbb{R} \times \mathbb{R} \times \mathbb{R} \times L^{1}((0,+\infty), \mathbb{R}) \times \mathbb{R} \times \mathbb{R}, \\
\chi^{+} & =\mathbb{R}^{+} \times \mathbb{R}^{+} \times \mathbb{R} \times L_{+}^{1}((0,+\infty), \mathbb{R}) \times \mathbb{R}^{+} \times \mathbb{R}^{+},
\end{aligned}
$$

and consider the linear operator $A: \operatorname{dom}(A) \subset \chi \rightarrow \chi$ defined by

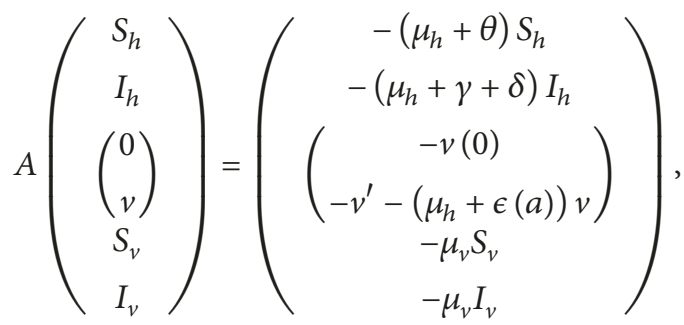


with $\operatorname{dom}(A)=\mathbb{R} \times \mathbb{R} \times\{0\} \times W^{1,1}((0,+\infty), \mathbb{R}) \times \mathbb{R} \times \mathbb{R}$, where $W^{1,1}$ is a Sobolev space. Then, $\overline{\operatorname{dom}(A)}=\mathbb{R} \times \mathbb{R} \times$ $\{0\} \times L^{1}((0,+\infty), \mathbb{R}) \times \mathbb{R} \times \mathbb{R}$ is not dense in $\chi$. We consider a nonlinear map $F: \overline{\operatorname{dom}(A)} \rightarrow \chi$ which is defined by

$$
\begin{aligned}
& F\left(\begin{array}{c}
S_{h} \\
I_{h} \\
0 \\
v
\end{array}\right) \\
& =\left(\begin{array}{c}
\Lambda_{h}-S_{h}(t) f\left(I_{v}(t)\right)+\int_{0}^{\infty} \epsilon(a) v(t, a) d a \\
S_{h}(t) f\left(I_{v}(t)\right) \\
\left(\begin{array}{c}
\theta S_{h}(t) \\
0_{L^{1}}
\end{array}\right) \\
\Lambda_{v}-S_{v}(t) g\left(I_{h}(t)\right) \\
S_{v}(t) g\left(I_{h}(t)\right)
\end{array}\right)
\end{aligned}
$$

and let

$$
u(t)=\left(S_{h}(t), I_{h}(t),\left(\begin{array}{c}
0 \\
v(t, \cdot)
\end{array}\right), S_{v}(t), I_{v}(t)\right)^{T} .
$$

Set

$$
\begin{aligned}
\chi_{0} & :=\overline{\operatorname{dom}(A)} \\
& =\mathbb{R} \times \mathbb{R} \times\{0\} \times L^{1}((0,+\infty), \mathbb{R}) \times \mathbb{R} \times \mathbb{R}, \\
\chi_{0}^{+} & :=\overline{\operatorname{dom}(A)} \cap \chi_{+} \\
& =\mathbb{R}^{+} \times \mathbb{R}^{+} \times\{0\} \times L_{+}^{1}((0,+\infty), \mathbb{R}) \times \mathbb{R}^{+} \times \mathbb{R}^{+} .
\end{aligned}
$$

Based on the above, we can reformulate system (3) as the following abstract Cauchy problem:

$$
\frac{d u(t)}{d t}=A u(t)+F u(t),
$$

$$
\text { for } t \geq 0 \text {, with } u(0) \in \chi_{0}^{+} \text {. }
$$

By applying the results given in $[19,21]$, we derive the existence and uniqueness of the semiflow $\{\Phi(t)\}_{t \geq 0}$ on $\chi_{0}^{+}$ generated by system (3). By using the theory for dynamical system (see [19]), we can further obtain the following lemma.

Lemma 1. System (3) generates a unique continuous semiflow $\{\Phi(t)\}_{t \geq 0}$ on $\chi_{0}^{+}$that is asymptotically smooth and bounded dissipative. Furthermore, the semiflow $\{\Phi(t)\}_{t \geq 0}$ has a compact global attractor $\mathscr{B} \subset \chi_{0}^{+}$.

The total population size of human hosts and vectors is, respectively,

$$
\begin{aligned}
& N_{h}(t)=S_{h}(t)+I_{h}(t)+\int_{0}^{\infty} v(t, a) d a, \\
& N_{v}(t)=S_{v}(t)+I_{v}(t) .
\end{aligned}
$$

Then, from the time derivative of $N_{h}(t)$ and $N_{v}(t)$, we get

$$
\begin{aligned}
& \frac{d N_{h}(t)}{d t} \leq \Lambda_{h}-\mu_{h} N_{h}(t), \\
& \frac{d N_{v}(t)}{d t}=\Lambda_{v}-\mu_{v} N_{v}(t),
\end{aligned}
$$

which implies

$$
\begin{aligned}
& \limsup _{t \rightarrow \infty} N_{h}(t) \leq \frac{\Lambda_{h}}{\mu_{h}}, \\
& \limsup _{t \rightarrow \infty} N_{v}(t) \leq \frac{\Lambda_{v}}{\mu_{v}} .
\end{aligned}
$$

We hence restrict our attention to solutions of (3) with initial conditions in

$$
\begin{aligned}
\Gamma & =\left\{\left(S_{h 0}, I_{h 0}, S_{v 0}, I_{v 0}, v_{0}(\cdot)\right) \in \mathbb{R}^{4+} \times L_{+}^{1}: v_{0}(0)\right. \\
& \left.=\theta S_{h 0}, N_{h}(0) \leq \frac{\Lambda_{h}}{\mu_{h}}, N_{v}(0) \leq \frac{\Lambda_{v}}{\mu_{v}}\right\} .
\end{aligned}
$$

The rest of the paper is structured as follows. In Section 2, we study the existence and local stability of equilibria of system (3). In Section 3, we present the results for the global dynamics of equilibria of system (3). In Section 4, the paper closes with conclusion.

\section{Existence and Local Stability of Equilibria}

In this part, we state the result about the existence and local stability of equilibria of the model (3). We first start by the existence of equilibria. We define

$$
\xi: \mathbb{R}^{+} \longrightarrow \mathbb{R}^{+},
$$

as

$$
\begin{aligned}
\xi(a) & =e^{-\mu_{h} a-\int_{0}^{a} \epsilon(s) d s}, \\
\mathscr{A} & =\int_{0}^{\infty} \epsilon(a) \xi(a) d a .
\end{aligned}
$$

Then,

$$
\mathscr{A} \leq \int_{0}^{\infty} \epsilon(a) e^{-\int_{0}^{a} \epsilon(s) d s} d a=1 .
$$

Let $\left(\bar{S}_{h}, \bar{I}_{h}, \bar{S}_{v}, \bar{I}_{v}, \bar{v}(\cdot)\right)$ be an equilibrium of (3). This implies

$$
\begin{aligned}
& \Lambda_{h}-\mu_{h} \bar{S}_{h}-\bar{S}_{h} f\left(\bar{I}_{v}\right)-\theta \bar{S}_{h}+\int_{0}^{\infty} \epsilon(a) \bar{v}(a) d a=0, \\
& \bar{S}_{h} f\left(\bar{I}_{v}\right)-\left(\mu_{h}+\gamma+\delta\right) \bar{I}_{h}=0, \\
& \frac{d \bar{v}(a)}{d a}=-\left(\mu_{h}+\epsilon(a)\right) \bar{v}(a), \\
& \Lambda_{v}-\mu_{v} \bar{S}_{v}-\bar{S}_{v} g\left(\bar{I}_{h}\right)=0, \\
& \bar{S}_{v} g\left(\bar{I}_{h}\right)-\mu_{v} \bar{I}_{v}=0 \\
& \bar{v}(0)=\theta \bar{S}_{h} .
\end{aligned}
$$


From the third and the sixth equations of (18), we deduce that

$$
\bar{v}(a)=\theta \bar{S}_{h} \xi(a) .
$$

By the first equation of (18), we get

$$
\bar{S}_{h}=\frac{\Lambda_{h}}{f\left(\bar{I}_{v}\right)+\mu_{h}+\theta(1-\mathscr{A})} .
$$

From the fourth equation of (18), we have

$$
\bar{S}_{v}=\frac{\Lambda_{v}}{g\left(\bar{I}_{h}\right)+\mu_{v}} .
$$

Substituting $\bar{S}_{h}$ and $\bar{S}_{v}$ into the second and the fifth equations of (18) gives

$$
\begin{aligned}
& \Lambda_{h} f\left(\bar{I}_{v}\right)-\left(\mu_{h}+\gamma+\delta\right)\left(\mu_{h}+\theta(1-\mathscr{A})+f\left(\bar{I}_{v}\right)\right) \bar{I}_{h} \\
& \quad=0 \\
& \Lambda_{v} g\left(\bar{I}_{h}\right)-\mu_{v} \bar{I}_{v}\left(\mu_{v}+g\left(\bar{I}_{h}\right)\right)=0 .
\end{aligned}
$$

From the second equation of (22), we obtain

$$
\bar{I}_{v}=\frac{\Lambda_{v} g\left(\bar{I}_{h}\right)}{\mu_{v}\left(\mu_{v}+g\left(\bar{I}_{h}\right)\right)} .
$$

Replacing $\bar{I}_{v}$ in the first equation of (22) yields

$$
\begin{aligned}
& \left(\Lambda_{h}-\mu_{h} \bar{I}_{h}\right) f\left(\frac{\Lambda_{v} g\left(\bar{I}_{h}\right)}{\mu_{v}\left(\mu_{v}+g\left(\bar{I}_{h}\right)\right)}\right) \\
& -\left(\mu_{h}+\gamma+\delta\right)\left(\mu_{h}+\theta(1-\mathscr{A})\right) \bar{I}_{h}=0 .
\end{aligned}
$$

By (H1) and (H2), $\bar{I}_{h}=0$ is a solution of the above equation. Thus, system (3) has a disease-free equilibrium

$$
\mathscr{E}_{0}=\left(\frac{\Lambda_{h}}{\mu_{h}+\theta(1-\mathscr{A})}, 0, \frac{\Lambda_{v}}{\mu_{v}}, 0, \frac{\theta \Lambda_{h} \xi(a)}{\mu_{h}+\theta(1-\mathscr{A})}\right) .
$$

Following the same method as [22], the basic reproduction number for model (3) is

$$
\mathscr{R}(\theta)=\sqrt{\frac{\Lambda_{h} \Lambda_{v} f^{\prime}(0) g^{\prime}(0)}{\mu_{v}^{2}\left(\mu_{h}+\gamma+\delta\right)\left(\mu_{h}+\theta(1-\mathscr{A})\right)}} .
$$

$\mathscr{R}(\theta)$ describes a threshold for endemic persistence/spread of the disease, the rate of increase in the number of cases during an epidemic. Its magnitude allows determining the effort necessary either to prevent an epidemic or to eliminate an infection from a population.

Let $\left(S_{h}^{*}, I_{h}^{*}, S_{v}^{*}, I_{v}^{*}, v^{*}(\cdot)\right)$ be an endemic equilibrium. Then, $I_{h}^{*} \in\left(0, \Lambda_{h} / \mu_{h}\right)$ and $h\left(I_{h}^{*}\right)=0$, where

$$
\begin{aligned}
h\left(I_{h}\right)= & \left(\Lambda_{h}-\mu_{h} I_{h}\right) f\left(\frac{\Lambda_{v} g\left(I_{h}\right)}{\mu_{v}\left(\mu_{v}+g\left(I_{h}\right)\right)}\right) \\
& -\left(\mu_{h}+\gamma+\delta\right)\left(\mu_{h}+\theta(1-\mathscr{A})\right) I_{h} .
\end{aligned}
$$

The function $h$ is continuous with $h(0)=0$ and $h\left(\Lambda_{h} / \mu_{h}\right) \leq 0$.
Moreover, for $I_{h} \in\left(0, \Lambda_{h} / \mu_{h}\right)$,

$$
\begin{aligned}
& \frac{d h}{d I_{h}} \\
& =\left(\Lambda_{h}-\mu_{h} I_{h}\right) \frac{\Lambda_{v} g^{\prime}\left(I_{h}\right)}{\left(\mu_{v}+g\left(I_{h}\right)\right)^{2}} f^{\prime}\left(\frac{\Lambda_{v} g\left(I_{h}\right)}{\mu_{v}\left(\mu_{v}+g\left(I_{h}\right)\right)}\right) \\
& \quad-\mu_{h} f\left(\frac{\Lambda_{v} g\left(I_{h}\right)}{\mu_{v}\left(\mu_{v}+g\left(I_{h}\right)\right)}\right) \\
& \quad-\left(\mu_{h}+\gamma+\delta\right)\left(\mu_{h}+\theta(1-\mathscr{A})\right) .
\end{aligned}
$$

The sufficient condition for $h$ to have a zero in $\left(0, \Lambda_{h} / \mu_{h}\right)$ is that $h$ is increasing at 0 . Thus, there is an endemic equilibrium if

$$
\begin{aligned}
\frac{d h}{d I_{h}}(0) & =\left(\mu_{h}+\gamma+\delta\right)\left(\mu_{h}+\theta(1-\mathscr{A})\right)\left(\mathscr{R}^{2}(\theta)-1\right) \\
& >0
\end{aligned}
$$

which is equivalent to $\mathscr{R}(\theta)>1$. Let $I_{h}^{*}$ be a unique solution in $\left(0, \Lambda_{h} / \mu_{h}\right)$ of $h\left(I_{h}\right)=0$. Then, system (3) admits a unique endemic equilibrium $\mathscr{E}^{*}=\left(S_{h}^{*}, I_{h}^{*}, S_{v}^{*}, I_{v}^{*}, v^{*}(\cdot)\right)$, where

$$
\begin{aligned}
& S_{h}^{*} \\
& \quad=\frac{\Lambda_{h}}{f\left(\Lambda_{v} g\left(I_{h}^{*}\right) / \mu_{v}\left(\mu_{v}+g\left(I_{h}^{*}\right)\right)\right)+\mu_{h}+\theta(1-\mathscr{A})}, \\
& S_{v}^{*}=\frac{\Lambda_{v}}{\mu_{v}+g\left(I_{h}^{*}\right)}, \\
& I_{v}^{*}=\frac{\Lambda_{v} g\left(I_{h}^{*}\right)}{\mu_{v}\left(\mu_{v}+g\left(I_{h}^{*}\right)\right)}, \\
& v^{*}(\cdot) \\
& \quad=\frac{\Lambda_{h} \theta \xi(a)}{f\left(\Lambda_{v} g\left(I_{h}^{*}\right) / \mu_{v}\left(\mu_{v}+g\left(I_{h}^{*}\right)\right)\right)+\mu_{h}+\theta(1-\mathscr{A})} .
\end{aligned}
$$

We summarize the above analysis in the following result.

Theorem 2 (consider system (3)). If $\mathscr{R}(\theta) \leq 1$, then there is a unique equilibrium, which is the disease-free equilibrium $\mathscr{E}_{0}$.

If $\mathscr{R}(\theta)>1$, then there are two equilibria, the disease-free equilibrium $\mathscr{E}_{0}$ and the endemic equilibrium $\mathscr{E}^{*}$.

We now deal with the local stability of the disease-free equilibrium. We show the stability of $\mathscr{E}_{0}$ by linearizing system (3) about $\mathscr{E}_{0}$. The result is stated as follows.

Theorem 3 (consider system (3)). If $\mathscr{R}(\theta)<1$, the diseasefree equilibrium $\mathscr{E}_{0}$ is locally asymptotically stable.

If $\mathscr{R}(\theta)>1$, the unique endemic equilibrium $\mathscr{E}^{*}$ is locally asymptotically stable. 
Proof. From the linearization of system (3) at $\mathscr{E}_{0}$, we deduce the following characteristic equation:

$$
\begin{aligned}
& \left(\lambda+\mu_{v}\right)\left(\lambda+\mu_{h}+\theta(1-\widehat{\mathscr{A}}(\lambda))\right) \\
& \cdot\left(\left(\lambda+\mu_{h}+\gamma+\delta\right)\left(\lambda+\mu_{v}\right)\right. \\
& \left.-\frac{\Lambda_{h}}{\mu_{h}+\theta(1-\mathscr{A})} \frac{\Lambda_{v}}{\mu_{v}} f^{\prime}(0) g^{\prime}(0)\right)=0,
\end{aligned}
$$

where

$$
\widehat{\mathscr{A}}(\lambda)=\int_{0}^{\infty} \epsilon(a) \xi(a) e^{-\lambda a} d a
$$

From (31), the eigenvalues are $-\mu_{v}$ and solutions of

$$
\begin{aligned}
\lambda+\mu_{h}+\theta & =\widehat{\theta \mathscr{A}}(\lambda) \\
\left(\lambda+\mu_{h}+\gamma+\delta\right)\left(\lambda+\mu_{v}\right) & =\frac{\Lambda_{h} \Lambda_{v} f^{\prime}(0) g^{\prime}(0)}{\mu_{v}\left(\mu_{h}+\theta(1-\mathscr{A})\right)} .
\end{aligned}
$$

All roots of (33) and (34) have negative real parts; otherwise let $\lambda_{0}$ be a root of (33) with $\operatorname{Re}\left(\lambda_{0}\right) \geq 0$. Then, we have

$$
\begin{aligned}
& \left|\lambda_{0}+\mu_{h}+\theta\right|>\theta, \\
& \left|\theta \widehat{\mathscr{A}}\left(\lambda_{0}\right)\right| \leq \theta \mathscr{A} \leq \theta .
\end{aligned}
$$

This leads to a contradiction.

Now, let $\lambda_{0}$ be a root of (34) with $\operatorname{Re}\left(\lambda_{0}\right) \geq 0$. From (26), we have

$$
\begin{aligned}
\left|\frac{\Lambda_{h} \Lambda_{v} f^{\prime}(0) g^{\prime}(0)}{\mu_{v}\left(\mu_{h}+\theta(1-\mathscr{A})\right)}\right| & =\mathscr{R}(\theta)^{2} \mu_{v}\left(\mu_{h}+\gamma+\delta\right) \\
& <\mu_{v}\left(\mu_{h}+\gamma+\delta\right) \\
& \leq\left|\left(\lambda_{0}+\mu_{h}+\gamma+\delta\right)\left(\lambda_{0}+\mu_{v}\right)\right| .
\end{aligned}
$$

This also leads to a contradiction by using (34) and then proves that $\mathscr{E}_{0}$ is locally asymptotically stable.

The characteristic equation at $\mathscr{E}^{*}$ is

$$
\begin{aligned}
& =\left|\begin{array}{cccc}
\lambda+\mu_{h}+f\left(I_{v}^{*}\right)+\theta(1-\widehat{\mathscr{A}}(\lambda)) & 0 & 0 & S_{h}^{*} f^{\prime}\left(I_{v}^{*}\right) \\
-f\left(I_{v}^{*}\right) & \lambda+\mu_{h}+\gamma+\delta & 0 & -S_{h}^{*} f^{\prime}\left(I_{v}^{*}\right) \\
0 & S_{v}^{*} g^{\prime}\left(I_{h}^{*}\right) & \lambda+\mu_{v}+g\left(I_{h}^{*}\right) & 0 \\
0 & -S_{v}^{*} g^{\prime}\left(I_{h}^{*}\right) & -g\left(I_{h}^{*}\right) & \lambda+\mu_{v}
\end{array}\right|, \\
0 & =\left(\lambda+\mu_{v}\right)\left[\left(\lambda+\mu_{h}+f\left(I_{v}^{*}\right)+\theta(1-\widehat{\mathscr{A}}(\lambda))\right)\left(\lambda+\mu_{h}+\gamma+\delta\right)\left(\lambda+\mu_{v}+g\left(I_{h}^{*}\right)\right)+S_{h}^{*} S_{v}^{*} f^{\prime}\left(I_{v}^{*}\right) f\left(I_{v}^{*}\right) g^{\prime}\left(I_{h}^{*}\right) g\left(I_{h}^{*}\right)\right. \\
& \left.-\left(\lambda+\mu_{h}+f\left(I_{v}^{*}\right)+\theta(1-\widehat{\mathscr{A}}(\lambda))\right) S_{h}^{*} S_{v}^{*} f^{\prime}\left(I_{v}^{*}\right) g^{\prime}\left(I_{h}^{*}\right)\right] .
\end{aligned}
$$

By using $S_{h}^{*}=\left(\mu_{h}+\gamma+\delta\right) I_{h}^{*} / f\left(I_{v}^{*}\right)$ and $S_{v}^{*}=\mu_{v} I_{v}^{*} / g\left(I_{h}^{*}\right)$, we get

$$
\begin{aligned}
0= & \left(\lambda+\mu_{v}\right)\left[\left(\lambda+\mu_{h}+f\left(I_{v}^{*}\right)+\theta(1-\widehat{\mathscr{A}}(\lambda))\right)\right. \\
& \cdot\left(\lambda+\mu_{h}+\gamma+\delta\right)\left(\lambda+\mu_{v}+g\left(I_{h}^{*}\right)\right) \\
& +\mu_{v}\left(\mu_{h}+\gamma+\delta\right) I_{h}^{*} I_{v}^{*} f^{\prime}\left(I_{v}^{*}\right) g^{\prime}\left(I_{h}^{*}\right) \\
& -\left(\lambda+\mu_{h}+f\left(I_{v}^{*}\right)+\theta(1-\widehat{\mathscr{A}}(\lambda))\right) \\
& \left.\times \frac{\mu_{v}\left(\mu_{h}+\gamma+\delta\right) I_{h}^{*} I_{v}^{*}}{f\left(I_{v}^{*}\right) g\left(I_{h}^{*}\right)} f^{\prime}\left(I_{v}^{*}\right) g^{\prime}\left(I_{h}^{*}\right)\right] .
\end{aligned}
$$

We show that the characteristic equation has no eigenvalues with nonnegative real parts. The eigenvalues are $-\mu_{v}$ and solutions of

$$
\begin{gathered}
\left(\lambda+\mu_{h}+f\left(I_{v}^{*}\right)+\theta(1-\widehat{\mathscr{A}}(\lambda))\right)\left(\lambda+\mu_{h}+\gamma+\delta\right) \\
\cdot\left(\lambda+\mu_{v}+g\left(I_{h}^{*}\right)\right)+\mu_{v}\left(\mu_{h}+\gamma+\delta\right) I_{h}^{*} I_{v}^{*} f^{\prime}\left(I_{v}^{*}\right) \\
\cdot g^{\prime}\left(I_{h}^{*}\right)=\left(\lambda+\mu_{h}+f\left(I_{v}^{*}\right)+\theta(1-\widehat{\mathscr{A}}(\lambda))\right) \\
\times \frac{\mu_{v}\left(\mu_{h}+\gamma+\delta\right) I_{h}^{*} I_{v}^{*}}{f\left(I_{v}^{*}\right) g\left(I_{h}^{*}\right)} f^{\prime}\left(I_{v}^{*}\right) g^{\prime}\left(I_{h}^{*}\right) .
\end{gathered}
$$

By way of contradiction, assume that there is one eigenvalue $\lambda_{1}$ with $\operatorname{Re}\left(\lambda_{1}\right) \geq 0$. Then,

$$
\begin{aligned}
& \left|1+\frac{\mu_{v}\left(\mu_{h}+\gamma+\delta\right) I_{h}^{*} I_{v}^{*} f^{\prime}\left(I_{v}^{*}\right) g^{\prime}\left(I_{h}^{*}\right)}{\left(\lambda_{1}+\mu_{h}+f\left(I_{v}^{*}\right)+\theta\left(1-\widehat{\mathscr{A}}\left(\lambda_{1}\right)\right)\right)\left(\lambda_{1}+\mu_{h}+\gamma+\delta\right)\left(\lambda_{1}+\mu_{v}+g\left(I_{h}^{*}\right)\right)}\right| \\
& \quad \times\left|\left(\lambda_{1}+\mu_{h}+\gamma+\delta\right)\left(\lambda_{1}+\mu_{v}+g\left(I_{h}^{*}\right)\right)\right|=\left|\frac{\mu_{v}\left(\mu_{h}+\gamma+\delta\right) I_{h}^{*} I_{v}^{*}}{f\left(I_{v}^{*}\right) g\left(I_{h}^{*}\right)} f^{\prime}\left(I_{v}^{*}\right) g^{\prime}\left(I_{h}^{*}\right)\right| .
\end{aligned}
$$


From (1), it follows that

$$
\leq \mu_{v}\left(\mu_{h}+\gamma+\delta\right)
$$

$$
\left|\frac{\mu_{v}\left(\mu_{h}+\gamma+\delta\right) I_{h}^{*} I_{v}^{*}}{f\left(I_{v}^{*}\right) g\left(I_{h}^{*}\right)} f^{\prime}\left(I_{v}^{*}\right) g^{\prime}\left(I_{h}^{*}\right)\right|
$$

Since

$$
\left|1+\frac{\mu_{v}\left(\mu_{h}+\gamma+\delta\right) I_{h}^{*} I_{v}^{*} f^{\prime}\left(I_{v}^{*}\right) g^{\prime}\left(I_{h}^{*}\right)}{\left(\lambda_{1}+\mu_{h}+f\left(I_{v}^{*}\right)+\theta\left(1-\widehat{\mathscr{A}}\left(\lambda_{1}\right)\right)\right)\left(\lambda_{1}+\mu_{h}+\gamma+\delta\right)\left(\lambda_{1}+\mu_{v}+g\left(I_{h}^{*}\right)\right)}\right|>1,
$$

we have

$$
\begin{aligned}
& \left|1+\frac{\mu_{v}\left(\mu_{h}+\gamma+\delta\right) I_{h}^{*} I_{v}^{*} f^{\prime}\left(I_{v}^{*}\right) g^{\prime}\left(I_{h}^{*}\right)}{\left(\lambda_{1}+\mu_{h}+f\left(I_{v}^{*}\right)+\theta\left(1-\widehat{\mathscr{A}}\left(\lambda_{1}\right)\right)\right)\left(\lambda_{1}+\mu_{h}+\gamma+\delta\right)\left(\lambda_{1}+\mu_{v}+g\left(I_{h}^{*}\right)\right)}\right| \\
& \quad \times\left|\left(\lambda_{1}+\mu_{h}+\gamma+\delta\right)\left(\lambda_{1}+\mu_{v}+g\left(I_{h}^{*}\right)\right)\right|>\mu_{v}\left(\mu_{h}+\gamma+\delta\right) .
\end{aligned}
$$

This leads to a contradiction.

\section{Global Stability Analysis of Equilibria}

In this section, we prove the global stability of the equilibria of model (3). We first start by the global stability of the diseasefree equilibrium $\mathscr{E}_{0}$. To attend this, we need the Fluctuation Lemma [23].

Let us introduce the notations

$$
\begin{aligned}
\psi_{\infty} & =\liminf _{t \rightarrow \infty} \psi(t), \\
\psi^{\infty} & =\limsup _{t \rightarrow \infty} \psi(t) .
\end{aligned}
$$

The Fluctuation Lemma is stated as follows.

Lemma 4 (See [23]). Let $\psi: \mathbb{R}^{+} \rightarrow \mathbb{R}$ be a bounded and continuously differentiable function. Then, there exist sequences $\left\{s_{n}\right\}$ and $\left\{t_{n}\right\}$ such that $s_{n} \rightarrow \infty, t_{n} \rightarrow \infty, \psi\left(s_{n}\right) \rightarrow \psi_{\infty}$, $\psi^{\prime}\left(s_{n}\right) \rightarrow 0, \psi\left(t_{n}\right) \rightarrow \psi^{\infty}$, and $\psi^{\prime}\left(t_{n}\right) \rightarrow 0$ as $n \rightarrow \infty$.

We also need the following lemma for establishing the global stability of $\mathscr{E}_{0}$.

Lemma 5 (See [18]). Suppose that $f: \mathbb{R}^{+} \rightarrow \mathbb{R}$ is a bounded function and $k \in L^{1}((0,+\infty), \mathbb{R})$. Then,

$$
\begin{aligned}
& \limsup _{t \rightarrow \infty} \int_{0}^{t} k(\zeta) f(t-\zeta) d \zeta \leq f^{\infty}\|k\|_{1} \\
& \text { where }\|k\|_{1}=\int_{0}^{+\infty} k(s) d s .
\end{aligned}
$$

We state the stability result of the disease-free equilibrium $\mathscr{E}_{0}$ as follows.

Theorem 6. If $\mathscr{R}(\theta)<1$, then the disease-free equilibrium $\mathscr{E}_{0}$ is globally asymptotically stable.
Proof. Using Theorem 3, it is sufficient to show that $\mathscr{E}_{0}$ is attractive in $\Gamma$.

Let $\left(S_{h}(t), I_{h}(t), S_{v}(t), I_{v}(t), v(t, a)\right)$ be a solution of (3) with $\left(S_{h 0}, I_{h 0}, S_{v 0}, I_{v 0}, v_{0}(\cdot)\right) \in \Gamma$. We integrate the third equation of (3) with the boundary conditions to obtain

$$
v(t, a)= \begin{cases}\theta S_{h}(t-a) \xi(a), & t \geq a, \\ v_{0}(a-t) \frac{\xi(a)}{\xi(a-t)}, & t<a .\end{cases}
$$

Using the Fluctuation Lemma 4, we derive

$$
\begin{aligned}
& S_{h}^{\infty} \leq \frac{\Lambda_{h}}{\mu_{h}+\theta(1-\mathscr{A})}, \\
& S_{v}^{\infty} \leq \frac{\Lambda_{v}}{\mu_{v}} .
\end{aligned}
$$

From (1) and (3), we get

$$
\begin{aligned}
\frac{d I_{h}(t)}{d t}= & S_{h}(t) f\left(I_{v}(t)\right)-\left(\mu_{h}+\gamma+\delta\right) I_{h} \\
\leq & \frac{\Lambda_{h}}{\mu_{h}+\theta(1-\mathscr{A})} f^{\prime}(0) I_{v}(t) \\
& -\left(\mu_{h}+\gamma+\delta\right) I_{h}(t), \\
\frac{d I_{v}(t)}{d t}= & S_{v}(t) g\left(I_{h}(t)\right)-\mu_{v} I_{v}(t) \\
\leq & \frac{\Lambda_{v}}{\mu_{v}} g^{\prime}(0) I_{h}(t)-\mu_{v} I_{v}(t) .
\end{aligned}
$$

From (48), we have

$$
\left(\begin{array}{l}
\frac{d I_{h}}{d t} \\
\frac{d I_{v}}{d t}
\end{array}\right)
$$




$$
\leq\left(\begin{array}{cc}
-\left(\mu_{h}+\gamma+\delta\right) & \frac{\Lambda_{h} f^{\prime}(0)}{\mu_{h}+\theta(1-\mathscr{A})} \\
\frac{\Lambda_{v} g^{\prime}(0)}{\mu_{v}} & -\mu_{v}
\end{array}\right)\left(\begin{array}{c}
I_{h} \\
I_{v}
\end{array}\right)
$$

It is evident that all eigenvalues of the matrix

$$
\left(\begin{array}{cc}
-\left(\mu_{h}+\gamma+\delta\right) & \frac{\Lambda_{h} f^{\prime}(0)}{\mu_{h}+\theta(1-\mathscr{A})} \\
\frac{\Lambda_{v} g^{\prime}(0)}{\mu_{v}} & -\mu_{v}
\end{array}\right)
$$

have negative real parts when $\mathscr{R}(\theta)<1$. This leads to

$$
\begin{aligned}
& I_{h}^{\infty} \longrightarrow 0, \\
& I_{v}^{\infty} \longrightarrow 0 .
\end{aligned}
$$

From Lemma 4, it follows that there exists a sequence $\left\{t_{n}\right\}$ such that $t_{n} \rightarrow \infty, S_{h}\left(t_{n}\right) \rightarrow S_{h, \infty}, S_{v}\left(t_{n}\right) \rightarrow S_{v, \infty}$, and $S_{h}^{\prime}\left(t_{n}\right) \rightarrow 0, S_{v}^{\prime}\left(t_{n}\right) \rightarrow 0$ as $n \rightarrow \infty$.

Note that

$$
\begin{aligned}
& \lim _{n \rightarrow \infty} I_{h}\left(t_{n}\right)=0, \\
& \lim _{n \rightarrow \infty} I_{v}\left(t_{n}\right)=0 .
\end{aligned}
$$

Thus,

$$
\begin{aligned}
\frac{d S_{h}\left(t_{n}\right)}{d t}= & \Lambda_{h}-\left(\mu_{h}+\theta\right) S_{h}\left(t_{n}\right)-S_{h}\left(t_{n}\right) f\left(I_{v}\left(t_{n}\right)\right) \\
& +\int_{0}^{t_{n}} \epsilon(a) S_{h}\left(t_{n}-a\right) \xi(a) d a \\
& +\int_{t_{n}}^{\infty} \epsilon(a) v_{0}\left(a-t_{n}\right) \frac{\xi(a)}{\xi\left(a-t_{n}\right)} d a, \\
\frac{d S_{v}\left(t_{n}\right)}{d t}= & \Lambda_{v}-\mu_{v} S_{v}\left(t_{n}\right)-S_{v}\left(t_{n}\right) g\left(I_{h}\left(t_{n}\right)\right) .
\end{aligned}
$$

Let $n \rightarrow \infty$; then

$$
\begin{gathered}
0 \geq \Lambda_{h}-\left(\mu_{h}+\theta\right) S_{h, \infty}-S_{h, \infty} f\left(I_{v}^{\infty}\right) \\
\quad+\int_{0}^{\infty} \epsilon(a) S_{h, \infty} \xi(a) d a, \\
0 \geq \Lambda_{v}-\mu_{v} S_{v, \infty}-S_{v, \infty} g\left(I_{h}^{\infty}\right),
\end{gathered}
$$

which gives

$$
\begin{aligned}
& 0 \geq \Lambda_{h}-\left(\mu_{h}+\theta(1-\mathscr{A})\right) S_{h, \infty}-S_{h, \infty} f\left(I_{v}^{\infty}\right), \\
& 0 \geq \Lambda_{v}-\mu_{v} S_{v, \infty}-S_{v, \infty} g\left(I_{h}^{\infty}\right) .
\end{aligned}
$$

Since $I_{h}^{\infty} \rightarrow 0$ and $I_{v}^{\infty} \rightarrow 0$, we obtain

$$
\begin{aligned}
\frac{\Lambda_{h}}{\mu_{h}+\theta(1-\mathscr{A})} & \leq S_{h, \infty} \leq S_{h}^{\infty} \leq \frac{\Lambda_{h}}{\mu_{h}+\theta(1-\mathscr{A})}, \\
\frac{\Lambda_{v}}{\mu_{v}} & \leq S_{v, \infty} \leq S_{v}^{\infty} \leq \frac{\Lambda_{v}}{\mu_{v}} .
\end{aligned}
$$

That is,

$$
\begin{aligned}
& \lim _{t \rightarrow \infty} S_{h}(t)=\frac{\Lambda_{h}}{\mu_{h}+\theta(1-\mathscr{A})}, \\
& \lim _{t \rightarrow \infty} S_{v}(t)=\frac{\Lambda_{v}}{\mu_{v}} .
\end{aligned}
$$

From (46), it follows that

$$
\lim _{t \rightarrow \infty} v(t, a)=\frac{\Lambda_{h} \theta \xi(a)}{\mu_{h}+\theta(1-\mathscr{A})} .
$$

Therefore, $\left(S_{h}(t), I_{h}(t), S_{v}(t), I_{v}(t), v(t, \cdot)\right) \rightarrow \mathscr{E}_{0}$ in $\mathbb{R}^{4+} \times L_{+}^{1}$ as $t \rightarrow \infty$.

We now deal with the global stability of the endemic equilibrium $\mathscr{E}^{*}$.

A total trajectory of $\Phi$ is a function $X: \mathbb{R} \rightarrow \mathbb{R}^{4+} \times L_{+}^{1}$ such that $\Phi(s, X(t))=X(t+s)$ for all $t \in \mathbb{R}$ and all $s \in \mathbb{R}^{+}$.

We define $\phi:(0, \infty) \rightarrow \mathbb{R}$ by $\phi(x)=x-1-\ln x . \phi$ has a strict global minimum at 1 with $\phi(1)=0$ and $\phi(x)>0$, $\forall x \neq 1$.

Lemma 7 (see [24]). Define

$$
F(X)=\phi\left(\frac{f(X)}{f\left(X^{*}\right)}\right)-\phi\left(\frac{X}{X^{*}}\right) .
$$

If assumptions (H1) and (H2) are satisfied, then $F(X) \leq 0$, $\forall X>0$.

The result of the global stability of the endemic equilibrium is stated as follows.

Theorem 8. If $\mathscr{R}(\theta)>1$, then the endemic equilibrium $\mathscr{E}^{*}$ is globally asymptotically stable in $\Gamma$.

Proof. Evaluating both sides of (3) at $\mathscr{E}_{*}$ gives

$$
\begin{aligned}
\Lambda_{h}+\int_{0}^{\infty} \epsilon(a) v^{*}(a) d a & =\left(\mu_{h}+f\left(I_{v}^{*}\right)+\theta\right) S_{h}^{*}, \\
S_{h}^{*} f\left(I_{v}^{*}\right) & =\left(\mu_{h}+\gamma+\delta\right) I_{h}^{*}, \\
\Lambda_{v} & =\left(\mu_{v}+g\left(I_{h}^{*}\right)\right) S_{v}^{*}, \\
g\left(I_{h}^{*}\right) S_{v}^{*} & =\mu_{v} I_{v}^{*}, \\
v^{*}(a) & =\theta S_{h}^{*} \xi(a) .
\end{aligned}
$$

Let

$$
\alpha(a)=\int_{a}^{\infty} \epsilon(s) v^{*}(s) d s
$$

Then,

$$
\frac{d \alpha(a)}{d a}=-\epsilon(a) v^{*}(a)
$$


Let

$$
\begin{aligned}
& V_{S_{h}}(t)=\phi\left(\frac{S_{h}(t)}{S_{h}^{*}}\right), \\
& V_{I_{h}}(t)=\phi\left(\frac{I_{h}(t)}{I_{h}^{*}}\right), \\
& V_{S_{v}}(t)=\phi\left(\frac{S_{v}(t)}{S_{v}^{*}}\right), \\
& V_{I_{v}}(t)=\phi\left(\frac{I_{v}(t)}{I_{v}^{*}}\right), \\
& V_{v}(t)=\int_{0}^{\infty} \alpha(a) \phi\left(\frac{v(t, a)}{v^{*}(a)}\right) .
\end{aligned}
$$

Define

$$
\begin{aligned}
V(t)= & S_{v}^{*} g\left(I_{h}^{*}\right)\left(S_{h}^{*} V_{S_{h}}(t)+I_{h}^{*} V_{I_{h}}(t)\right) \\
& +S_{h}^{*} f\left(I_{v}^{*}\right)\left(S_{v}^{*} V_{S_{v}}(t)+I_{v}^{*} V_{I_{v}}(t)\right) \\
& +S_{v}^{*} g\left(I_{h}^{*}\right) V_{v}(t) .
\end{aligned}
$$

We study the behavior of the Lyapunov functional $V(t)$ given by (68). $V(t)$ is bounded and $V(t) \geq 0$ with equality if and only if $S_{h}(t) / S_{h}^{*}=I_{h}(t) / I_{h}^{*}=S_{v}(t) / S_{v}^{*}=I_{v}(t) / I_{v}^{*}=v(t, a) /$ $v^{*}(a)=1$.

For clarity, the derivatives of $V_{S_{h}}(t), V_{I_{h}}(t), V_{S_{v}}(t), V_{I_{v}}(t)$, $V_{v}(t)$ will be calculated separately and then combined to obtain $d V(t) / d t$. We first have

$$
\begin{aligned}
& \frac{d V_{S_{h}}(t)}{d t}=\frac{1}{S_{h}^{*}}\left(1-\frac{S_{h}^{*}}{S_{h}(t)}\right) \frac{d S_{h}(t)}{d t}=\frac{1}{S_{h}^{*}}(1 \\
& \left.-\frac{S_{h}^{*}}{S_{h}(t)}\right)\left(\Lambda_{h}-\mu_{h} S_{h}(t)-S_{h}(t) f\left(I_{v}(t)\right)\right. \\
& \left.-\theta S_{h}(t)+\int_{0}^{\infty} \epsilon(a) v(t, a) d a\right) .
\end{aligned}
$$

Using (60) to replace $\Lambda_{h}$ in (69) gives

$$
\begin{gathered}
\frac{d V_{S_{h}}(t)}{d t}=\frac{1}{S_{h}^{*}}\left(1-\frac{S_{h}^{*}}{S_{h}(t)}\right)\left(\left(\mu_{h}+\theta\right) S_{h}^{*}+S_{h}^{*} f\left(I_{v}^{*}\right)\right. \\
-\int_{0}^{\infty} \epsilon(a) v^{*}(a) d a-\left(\mu_{h}+\theta\right) S_{h}(t)-S_{h}(t) \\
\left.\cdot f\left(I_{v}(t)\right)+\int_{0}^{\infty} \epsilon(a) v(t, a) d a\right)=-\left(\mu_{h}+\theta\right) \\
\cdot \frac{\left(S_{h}(t)-S_{h}^{*}\right)^{2}}{S_{h}(t) S_{h}^{*}}+f\left(I_{v}^{*}\right)\left(1-\frac{S_{h}(t) f\left(I_{v}(t)\right)}{S_{h}^{*} f\left(I_{v}^{*}\right)}\right. \\
\left.-\frac{S_{h}^{*}}{S_{h}(t)}+\frac{f\left(I_{v}(t)\right)}{f\left(I_{v}^{*}\right)}\right)+\int_{0}^{\infty} \frac{\epsilon(a) v^{*}(a)}{S_{h}^{*}}\left(\frac{v(t, a)}{v^{*}(a)}\right. \\
\left.-\frac{S_{h}^{*} v(t, a)}{S_{h}(t) v^{*}(a)}-1+\frac{S_{h}^{*}}{S_{h}(t)}\right) d a .
\end{gathered}
$$

Next, we calculate $d V_{I_{h}}(t) / d t$.

$$
\begin{aligned}
& \frac{d V_{I_{h}}(t)}{d t}=\frac{1}{I_{h}^{*}}\left(1-\frac{I_{h}^{*}}{I_{h}(t)}\right) \frac{d I_{h}}{d t}=\frac{1}{I_{h}^{*}}\left(1-\frac{I_{h}^{*}}{I_{h}(t)}\right) \\
& \cdot\left(S_{h}(t) f\left(I_{v}(t)\right)-\left(\mu_{h}+\gamma+\delta\right) I_{h}(t)\right) \\
& =\frac{1}{I_{h}^{*}}\left(1-\frac{I_{h}^{*}}{I_{h}(t)}\right) \\
& \cdot\left(S_{h}(t) f\left(I_{v}(t)\right)-\left(\mu_{h}+\gamma+\delta\right) I_{h}^{*} \frac{I_{h}(t)}{I_{h}^{*}}\right) .
\end{aligned}
$$

Using (61) to replace $\left(\mu_{h}+\gamma+\delta\right) I_{h}^{*}$ in (71) gives

$$
\begin{aligned}
& \frac{d V_{I_{h}}(t)}{d t}=\frac{1}{I_{h}^{*}}\left(1-\frac{I_{h}^{*}}{I_{h}(t)}\right)\left(S_{h}(t) f\left(I_{v}(t)\right)\right. \\
& \left.-S_{h}^{*} f\left(I_{v}^{*}\right) \frac{I_{h}(t)}{I_{h}^{*}}\right)=\frac{S_{h}^{*} f\left(I_{v}^{*}\right)}{I_{h}^{*}}(1 \\
& \left.\quad+\frac{S_{h}(t) f\left(I_{v}(t)\right)}{S_{h}^{*} f\left(I_{v}^{*}\right)}-\frac{S_{h}(t) f\left(I_{v}(t)\right) I_{h}^{*}}{S_{h}^{*} f\left(I_{v}^{*}\right) I_{h}(t)}-\frac{I_{h}(t)}{I_{h}^{*}}\right) .
\end{aligned}
$$

We now calculate the derivative of $V_{S_{v}(t)}$.

$$
\begin{aligned}
& \frac{d V_{S_{v}}(t)}{d t}=\frac{1}{S_{v}^{*}}\left(1-\frac{S_{v}^{*}}{S_{v}(t)}\right) \frac{d S_{v}}{d t} \\
& =\frac{1}{S_{v}^{*}}\left(1-\frac{S_{v}^{*}}{S_{v}(t)}\right)\left(\Lambda_{v}-\mu_{v} S_{v}(t)-S_{v}(t) g\left(I_{h}(t)\right)\right) .
\end{aligned}
$$

Using (62) to replace $\Lambda_{v}$ in (73) gives

$$
\begin{gathered}
\frac{d V_{S_{v}}(t)}{d t}=\frac{1}{S_{v}^{*}}\left(1-\frac{S_{v}^{*}}{S_{v}(t)}\right) \frac{d S_{v}}{d t}=\frac{1}{S_{v}^{*}}\left(1-\frac{S_{v}^{*}}{S_{v}(t)}\right) \\
\cdot\left(\mu S_{v}^{*}+S_{v}^{*} g\left(I_{h}^{*}\right)-\mu_{v} S_{v}(t)-S_{v}(t) g\left(I_{h}(t)\right)\right) \\
=-\mu_{v} \frac{\left(S_{v}(t)-S_{v}^{*}\right)^{2}}{S_{v}(t) S_{v}^{*}}+g\left(I_{h}^{*}\right) \\
\cdot\left(1-\frac{S_{v}(t) g\left(I_{h}(t)\right)}{S_{v}^{*} g\left(I_{h}^{*}\right)}-\frac{S_{v}^{*}}{S_{v}(t)}+\frac{g\left(I_{h}(t)\right)}{g\left(I_{h}^{*}\right)}\right) .
\end{gathered}
$$

Differentiating $V_{I_{v}}(t)$ with respect to $t$ yields

$$
\begin{aligned}
& \frac{d V_{I_{v}}(t)}{d t}=\frac{1}{I_{v}^{*}}\left(1-\frac{I_{v}^{*}}{I_{v}(t)}\right) \frac{d I_{v}}{d t} \\
& =\frac{1}{I_{v}^{*}}\left(1-\frac{I_{v}^{*}}{I_{v}(t)}\right)\left(S_{v}(t) g\left(I_{h}(t)\right)-\mu_{v} I_{v}(t)\right) \\
& =\frac{1}{I_{v}^{*}}\left(1-\frac{I_{v}^{*}}{I_{v}(t)}\right)\left(S_{v}(t) g\left(I_{h}(t)\right)-\mu_{v} I_{v}^{*} \frac{I_{v}(t)}{I_{v}^{*}}\right) .
\end{aligned}
$$


Using (63) to replace $\mu_{v} I_{v}^{*}$ in (75) gives

$$
\begin{aligned}
& \frac{d V_{I_{v}}(t)}{d t}=\frac{1}{I_{v}^{*}}\left(1-\frac{I_{v}^{*}}{I_{v}(t)}\right)\left(S_{v}(t) g\left(I_{h}(t)\right)\right. \\
& \left.-S_{v}^{*} g\left(I_{h}^{*}\right) \frac{I_{v}(t)}{I_{v}^{*}}\right)=\frac{S_{v}^{*} g\left(I_{h}^{*}\right)}{I_{v}^{*}}(1 \\
& \left.+\frac{S_{v}(t) g\left(I_{h}(t)\right)}{S_{v}^{*} g\left(I_{h}^{*}\right)}-\frac{S_{v}(t) g\left(I_{h}(t)\right) I_{v}^{*}}{S_{v}^{*} g\left(I_{h}^{*}\right) I_{v}(t)}-\frac{I_{v}(t)}{I_{v}^{*}}\right) .
\end{aligned}
$$

The derivative of $V_{v}(t)$ is

$$
\begin{aligned}
& \frac{d V_{v}(t)}{d t}=\int_{0}^{\infty} \alpha(a) \frac{\partial \phi\left(v(t, a) / v^{*}(a)\right)}{\partial t} d a=\int_{0}^{\infty} \alpha(a) \\
& \cdot\left(1-\frac{v^{*}(a)}{v(t, a)}\right) \frac{1}{v^{*}(a)} \frac{\partial v(t, a)}{\partial t} d a=-\int_{0}^{\infty} \alpha(a) \\
& \cdot\left(1-\frac{v^{*}(a)}{v(t, a)}\right) \frac{v(t, a)}{v^{*}(a)}\left(\frac{v_{a}(t, a)}{v(t, a)}+\mu_{h}+\epsilon(a)\right) d a \\
& =-\int_{0}^{\infty} \alpha(a)\left(\frac{v(t, a)}{v^{*}(a)}-1\right) \\
& \cdot\left(\frac{v_{a}(t, a)}{v(t, a)}+\mu_{h}+\epsilon(a)\right) d a,
\end{aligned}
$$

where $v_{a}(t, a)=\partial v(t, a) / \partial a$.

Using $(\partial / \partial a) \phi\left(v(t, a) / v^{*}(a)\right)=\left(v(t, a) / v^{*}(a)-1\right)\left(v_{a}(t\right.$, $\left.a) / v(t, a)+\mu_{h}+\epsilon(a)\right), d \alpha(a) / d a=-\epsilon(a) v^{*}(a)$ and integration by parts, we get

$$
\begin{aligned}
\frac{d V_{v}(t)}{d t}= & -\int_{0}^{\infty} \alpha(a) \frac{\partial}{\partial a} \phi\left(\frac{v(t, a)}{v^{*}(a)}\right) d a \\
= & -\left.\alpha(a) \phi\left(\frac{v(t, a)}{v^{*}(a)}\right)\right|_{a=0} ^{a=\infty} \\
& +\int_{0}^{\infty} \phi\left(\frac{v(t, a)}{v^{*}(a)}\right) \frac{d \alpha(a)}{d a} d a \\
= & -\left.\alpha(a) \phi\left(\frac{v(t, a)}{v^{*}(a)}\right)\right|_{a=\infty} \\
& +\alpha(0) \phi\left(\frac{v(t, 0)}{v^{*}(0)}\right) \\
& -\int_{0}^{\infty} \epsilon(a) v^{*}(a) \phi\left(\frac{v(t, a)}{v^{*}(a)}\right) d a .
\end{aligned}
$$

From $\alpha(0)=\int_{0}^{\infty} \epsilon(a) v^{*}(a) d a$, we get

$$
\begin{aligned}
\frac{d V_{v}(t)}{d t}= & -\left.\alpha(a) \phi\left(\frac{v(t, a)}{v^{*}(a)}\right)\right|_{a=\infty} \\
& +\int_{0}^{\infty} \epsilon(a) v^{*}(a) \phi\left(\frac{v(t, 0)}{v^{*}(0)}\right) d a \\
& -\int_{0}^{\infty} \epsilon(a) v^{*}(a) \phi\left(\frac{v(t, a)}{v^{*}(a)}\right) d a .
\end{aligned}
$$

Combining (70), (72), (74), (76), and (79) and multiplying appropriately by coefficients determined by (68), we obtain

$$
\begin{aligned}
& \frac{d V(t)}{d t}=-\left(\mu_{h}+\theta\right) S_{v}^{*} g\left(I_{h}^{*}\right) \frac{\left(S_{h}-S_{h}^{*}\right)^{2}}{S_{h}(t)} \\
& -\mu_{v} S_{h}^{*} f\left(I_{v}^{*}\right) \frac{\left(S_{v}-S_{v}^{*}\right)^{2}}{S_{v}(t)}+S_{h}^{*} S_{v}^{*} f\left(I_{v}^{*}\right) g\left(I_{h}^{*}\right)(4 \\
& -\frac{S_{h}(t) f\left(I_{v}(t)\right) I_{h}^{*}}{S_{h}^{*} f\left(I_{v}^{*}\right) I_{h}(t)}-\frac{S_{h}^{*}}{S_{h}(t)}+\frac{f\left(I_{v}(t)\right)}{f\left(I_{v}^{*}\right)}-\frac{I_{h}(t)}{I_{h}^{*}} \\
& -\frac{I_{v}(t)}{I_{v}^{*}}-\frac{S_{v}(t) g\left(I_{h}(t)\right) I_{v}^{*}}{S_{v}^{*} g\left(I_{h}^{*}\right) I_{v}(t)}-\frac{S_{v}^{*}}{S_{v}(t)} \\
& \left.+\frac{g\left(I_{h}(t)\right)}{g\left(I_{h}^{*}\right)}\right)-\left.\alpha(a) S_{v}^{*} g\left(I_{h}^{*}\right) \phi\left(\frac{v(t, a)}{v^{*}(a)}\right)\right|_{a=\infty} \\
& +S_{v}^{*} g\left(I_{h}^{*}\right) \int_{0}^{\infty} \epsilon(a) v^{*}(a)\left(\phi\left(\frac{v(t, 0)}{v^{*}(0)}\right)\right. \\
& -\phi\left(\frac{v(t, a)}{v^{*}(a)}\right)+\frac{v(t, a)}{v^{*}(a)}-\frac{S_{h}^{*} v(t, a)}{S_{h}(t) v^{*}(a)}-1 \\
& \left.+\frac{S_{h}^{*}}{S_{h}(t)}\right) d a \text {. }
\end{aligned}
$$

By $\int_{0}^{\infty} \epsilon(a) v^{*}(a) d a=\theta S_{h}^{*} K, v(t, 0)=\theta S_{h}(t)$, and $v^{*}(0)=$ $\theta S_{h}^{*}$, it follows that

$$
\begin{aligned}
& \frac{d V(t)}{d t}=-\left(\mu_{h}+\theta(1-K)\right) S_{v}^{*} g\left(I_{h}^{*}\right) \frac{\left(S_{h}-S_{h}^{*}\right)^{2}}{S_{h}(t)} \\
& -\mu_{v} S_{h}^{*} f\left(I_{v}^{*}\right) \frac{\left(S_{v}-S_{v}^{*}\right)^{2}}{S_{v}(t)}+S_{h}^{*} S_{v}^{*} f\left(I_{v}^{*}\right) g\left(I_{h}^{*}\right) \\
& \cdot\left(\phi\left(\frac{f\left(I_{v}(t)\right)}{f\left(I_{v}^{*}\right)}\right)-\phi\left(\frac{I_{v}(t)}{I_{v}^{*}}\right)+\phi\left(\frac{g\left(I_{h}(t)\right)}{g\left(I_{h}^{*}\right)}\right)\right. \\
& -\phi\left(\frac{I_{h}(t)}{I_{h}^{*}}\right)-\phi\left(\frac{S_{v}^{*}}{S_{v}(t)}\right) \\
& \left.-\phi\left(\frac{S_{v}(t) g\left(I_{h}(t)\right) I_{v}^{*}}{S_{v}^{*} g\left(I_{h}^{*}\right) I_{v}(t)}\right)\right) \\
& -\left.\alpha(a) S_{v}^{*} g\left(I_{h}^{*}\right) \phi\left(\frac{v(t, a)}{v^{*}(a)}\right)\right|_{a=\infty}-S_{v}^{*} g\left(I_{h}^{*}\right) \\
& \cdot \int_{0}^{\infty} \epsilon(a) v^{*}(a) \phi\left(\frac{S_{h}^{*} v(t, a)}{S_{h}(t) v^{*}(a)}\right) .
\end{aligned}
$$

Thus, from Lemma 7, we deduce that

$$
\frac{d V(t)}{d t} \leq 0
$$

that is, $V$ is nonincreasing. Denote by $\mathscr{M}$ the largest invariant subset of $\{d V(t) / d t=0\}$. 
Since $V$ is bounded on $X(\cdot)$, the $\omega$-limit set of $X(\cdot)$ must be contained in $\mathscr{M}$. $v^{*}(a)$.

$d V(t) / d t=0$ yields $S_{h}(t)=S_{h}^{*}, S_{v}(t)=S_{v}^{*}$, and $v(t, a)=$

Thus, $d S_{h}(t) / d t=d S_{v}(t) / d t=0$ in $\mathscr{M}$. This implies that

$$
\begin{aligned}
& \Lambda_{h}-\left(\mu_{h}+\theta\right) S_{h}^{*}-S_{h}^{*} f\left(I_{v}(t)\right)+\int_{0}^{\infty} \epsilon(a) v^{*}(a) d a \\
& \quad=0, \\
& \Lambda_{v}-\mu_{v} S_{v}^{*}-S_{v}^{*} g\left(I_{h}(t)\right)=0,
\end{aligned}
$$

for $t \in \mathbb{R}$, which gives $f\left(I_{v}(t)\right)=f\left(I_{v}^{*}\right)$ and $g\left(I_{h}(t)\right)=g\left(I_{h}^{*}\right)$ $\forall t \in \mathbb{R}$. The monotonicity of $f$ and $g$ stated in (H1) and (H2) implies that $I_{v}(t)=I_{v}^{*}$ and $I_{h}(t)=I_{h}^{*}, \forall t \in \mathbb{R}$. Therefore, $\mathscr{M}=\left\{\mathscr{E}^{*}\right\}$.

Then, the $\omega$-limit set of $X(\cdot)$ is the endemic equilibrium $\mathscr{E}^{*}$ and hence $V(X(t)) \geq V\left(\mathscr{E}^{*}\right), \forall t \in \mathbb{R}$. Thus, $\mathscr{B}=\left\{\mathscr{E}^{*}\right\}$.

\section{Conclusion}

We have analysed a vector-borne disease model with nonlinear incidences, in which we have incorporated the waning of vaccine-induced immunity. These nonlinear incidences rates include mass action and saturating incidence as special cases. The basic reproduction number denoted by $\mathscr{R}(\theta)$ is derived. The model exhibits two equilibria, namely, the disease-free equilibrium $\mathscr{E}_{0}$ and the endemic equilibrium $\mathscr{E}^{*}$. We have shown that if $\mathscr{R}(\theta)$ is less than 1 , then the disease-free equilibrium $\mathscr{E}_{0}$ is globally asymptotically stable; that is, the disease dies out and if $\mathscr{R}(\theta)$ is larger than 1, then the endemic equilibrium $\mathscr{E}^{*}$ is globally asymptotically stable; that is, the disease persists in the population.

From these results, a critical vaccine coverage rate is obtained by solving the equation $\mathscr{R}(\theta)=1$, which yields

$$
\begin{aligned}
& \theta_{0} \\
& =\frac{1}{1-\mathscr{A}}\left(\frac{\Lambda_{h} \Lambda_{v} f^{\prime}(0) g^{\prime}(0)-\mu_{h} \mu_{v}^{2}\left(\mu_{h}+\gamma+\delta\right)}{\mu_{v}^{2}\left(\mu_{h}+\gamma+\delta\right)}\right) .
\end{aligned}
$$

Then, if the vaccine coverage rate $\theta$ is greater than $\theta_{0}$, then $\mathscr{R}(\theta)<1$ and the disease will die out. The critical vaccine coverage rate $\theta_{0}$ is increasing in the waning of vaccine. Then, neglecting the waning of vaccine (i.e., $\mathscr{A}=0$ ) when applying a vaccination for vector-borne disease will surely not be sufficient to make the disease die out in the population.

\section{Conflicts of Interest}

The authors declare that there are no conflicts of interest regarding the publication of this paper.

\section{References}

[1] R. Ross, The prevention of Malaria, John Murray, London, UK, 1911.

[2] M. Haber, I. M. Longini, and M. E. Halloran, "Measures of the effects of vaccination in a randomly mixing population,"
International Journal of Epidemiology, vol. 20, no. 1, pp. 300-310, 1991.

[3] M. Iannelli, M. Martcheva, and X.-Z. Li, "Strain replacement in an epidemic model with super-infection and perfect vaccination," Mathematical Biosciences, vol. 195, no. 1, pp. 23-46, 2005.

[4] I. V. Coutinho-Abreu and M. Ramalho-Ortigao, "Transmission blocking vaccines to control insect-borne diseases - A review," Memórias do Instituto Oswaldo Cruz, vol. 105, no. 1, pp. 1-12, 2010.

[5] B. Shulgin, L. Stone, and Z. Agur, "Pulse vaccination strategy in the SIR epidemic model," Bulletin of Mathematical Biology, vol. 60, no. 6, pp. 1123-1148, 1998.

[6] L. Cai, X. Li, M. Ghosh, and B. Guo, "Stability analysis of an HIV/AIDS epidemic model with treatment," Journal of Computational and Applied Mathematics, vol. 229, no. 1, pp. 313323, 2009.

[7] X.-Z. Li, J. Wang, and M. Ghosh, "Stability and bifurcation of an SIVS epidemic model with treatment and age of vaccination," Applied Mathematical Modelling, vol. 34, no. 2, pp. 437-450, 2010.

[8] J. Yang, M. Martcheva, and L. Wang, "Global threshold dynamics of an SIVS model with waning vaccine-induced immunity and nonlinear incidence," Mathematical Biosciences, vol. 268, pp. 1-8, 2015.

[9] J. S. A. Linda, "An introduction to mathematical Biology", in USA, pp. 133-127, Pearson Education Ltd, 2007.

[10] R. E. Mickens, "A discrete-time model for the spread of periodic diseases without immunity," BioSystems, vol. 26, no. 3, pp. 193198, 1992.

[11] X. Liu, Y. Takeuchi, and S. Iwami, "SVIR epidemic models with vaccination strategies," Journal of Theoretical Biology, vol. 253, no. 1, pp. 1-11, 2008.

[12] Y. Xiao and S. Tang, "Dynamics of infection with nonlinear incidence in a simple vaccination model," Nonlinear Analysis: Real World Applications, vol. 11, no. 5, pp. 4154-4163, 2010.

[13] A. B. Gumel and S. M. Moghadas, "A qualitative study of a vaccination model with non-linear incidence," Applied Mathematics and Computation, vol. 143, no. 2-3, pp. 409-419, 2003.

[14] S. Gao, L. Chen, J. J. Nieto, and A. Torres, "Analysis of a delayed epidemic model with pulse vaccination and saturation incidence," Vaccine, vol. 24, no. 35-36, pp. 6037-6045, 2006.

[15] J. R. Kremer, F. Schneider, and C. P. Muller, "Waning antibodies in measles and rubella vaccinees - A longitudinal study," Vaccine, vol. 24, no. 14, pp. 2594-2601, 2006.

[16] F. R. Mooi, N. A. van der Maas, and H. E. de Melker, "Pertussis resurgence: waning immunity and pathogen adaptation-two sides of the same coin," Epidemiology and Infection, vol. 142, no. 4, pp. 685-694, 2014.

[17] A. M. Wendelboe, A. Van Rie, S. Salmaso, and J. A. Englund, "Duration of immunity against pertussis after natural infection or vaccination," The Pediatric Infectious Disease Journal, vol. 24, no. 5, pp. S58-S61, 2005.

[18] M. Iannelli, "Mathematical theory of age-structured population dynamics, in applied mathematics monographs 7," in In Proceedings of the Comitato Nazionale per le Sciencze Mathematiche, Consiglio Nazionale dell Ricerche (C.N.R), Giardini, Italy, 1995.

[19] P. Magal, "Compact attractors for time-periodic age-structured population models," Electronic Journal of Differential Equations, No. 65, 35 pages, 2001.

[20] C. J. Browne and S. S. Pilyugin, "Global analysis of agestructured within-host virus model," Discrete and Continuous Dynamical Systems - Series B, vol. 18, no. 8, pp. 1999-2017, 2013. 
[21] H. R. Thieme, "Semiflows generated by Lipschitz perturbations of non-densely defined operators," Differential and Integral Equations: International Journal for Theory and Applications, vol. 3, no. 6, pp. 1035-1066, 1990.

[22] O. Diekmann, J. A. Heesterbeek, and J. A. Metz, "On the definition and the computation of the basic reproduction ratio $R_{0}$ in models for infectious diseases in heterogeneous populations," Journal of Mathematical Biology, vol. 28, no. 4, pp. 365-382, 1990.

[23] W. M. Hirsch, H. Hanisch, and J.-P. Gabriel, "Differential equation models of some parasitic infections: methods for the study of asymptotic behavior," Communications on Pure and Applied Mathematics, vol. 38, no. 6, pp. 733-753, 1985.

[24] R. P. Sigdel and C. C. McCluskey, "Global stability for an it SEI model of infectious disease with immigration," Applied Mathematics and Computation, vol. 243, pp. 684-689, 2014. 


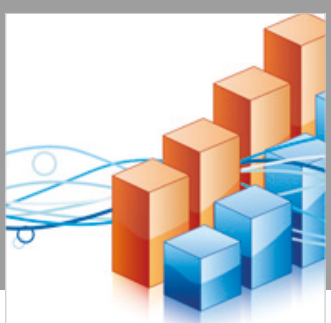

Advances in

Operations Research

\section{-n-m}
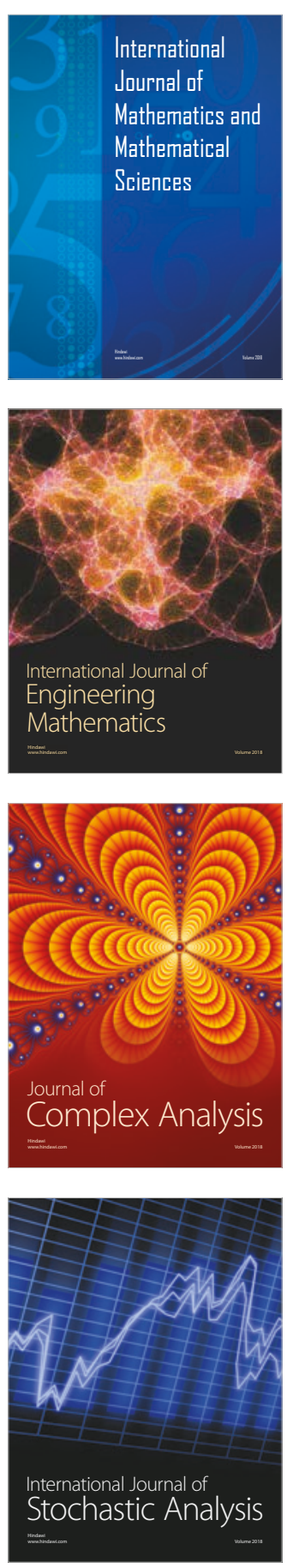
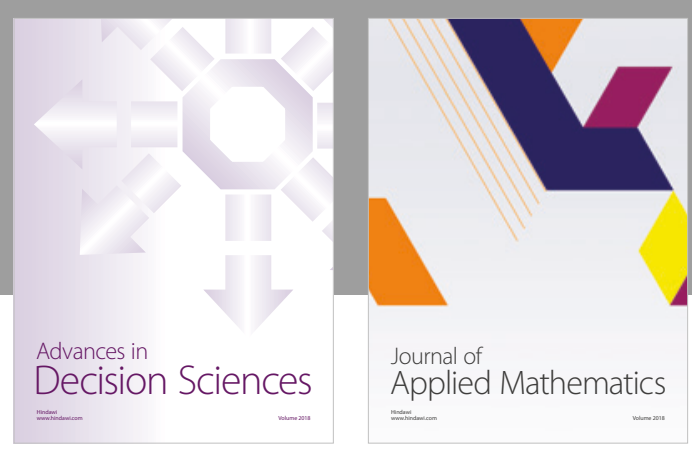

Journal of

Applied Mathematics
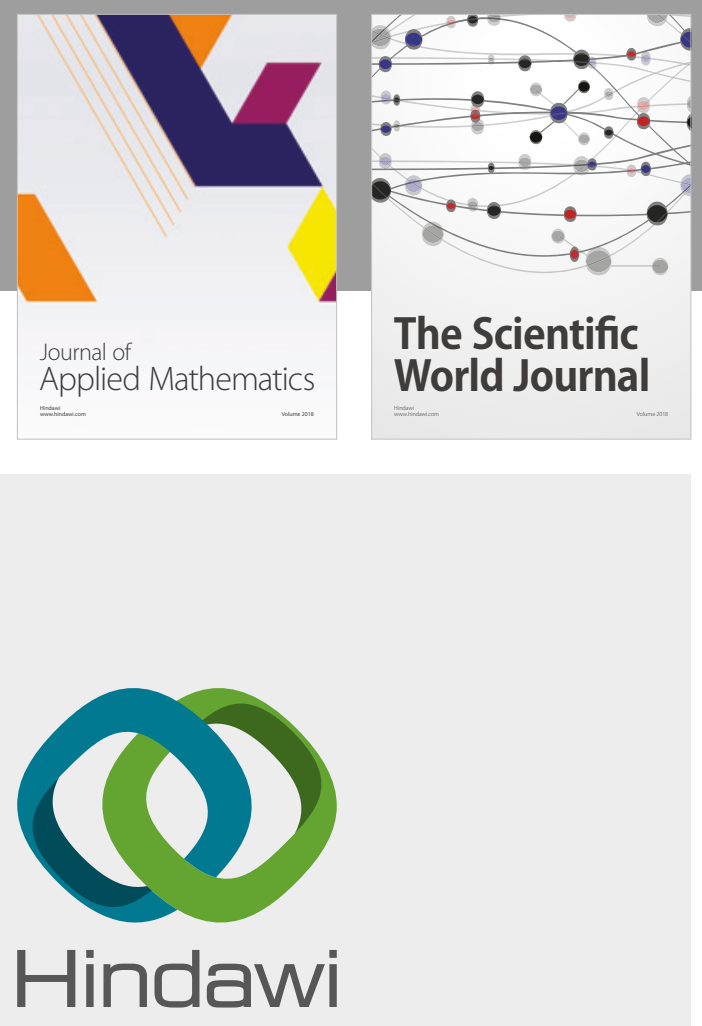

Submit your manuscripts at

www.hindawi.com

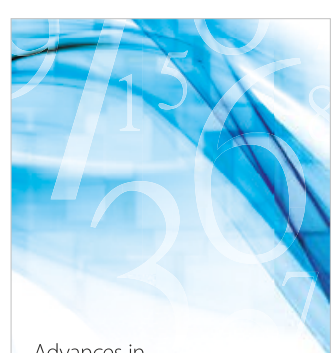

Advances in
Numerical Analysis
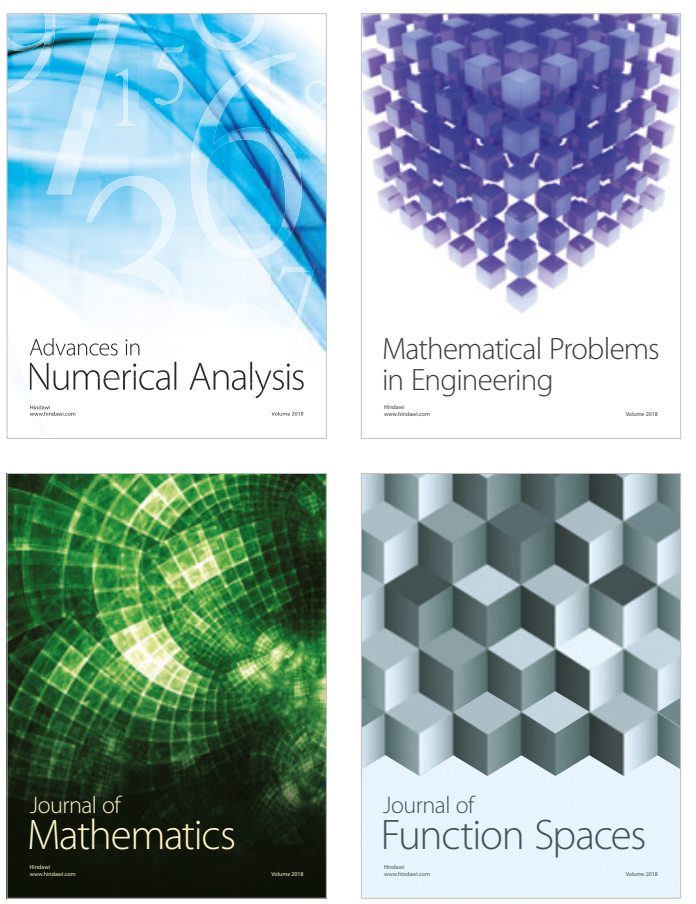

Mathematical Problems in Engineering

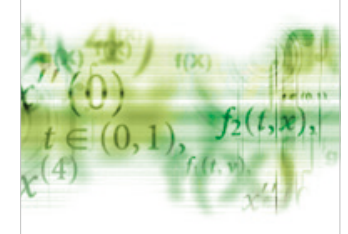

International Journal of

Differential Equations

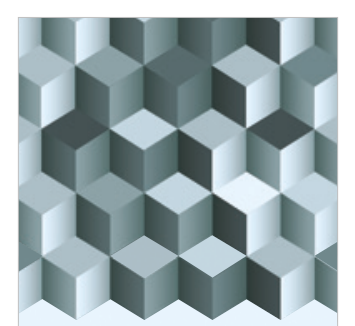

Journal of

Function Spaces

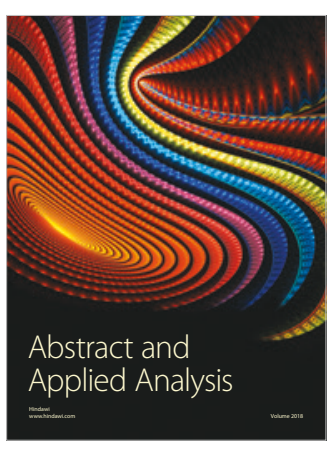

The Scientific

World Journal

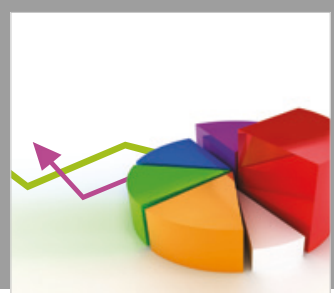

Journal of

Probability and Statistics
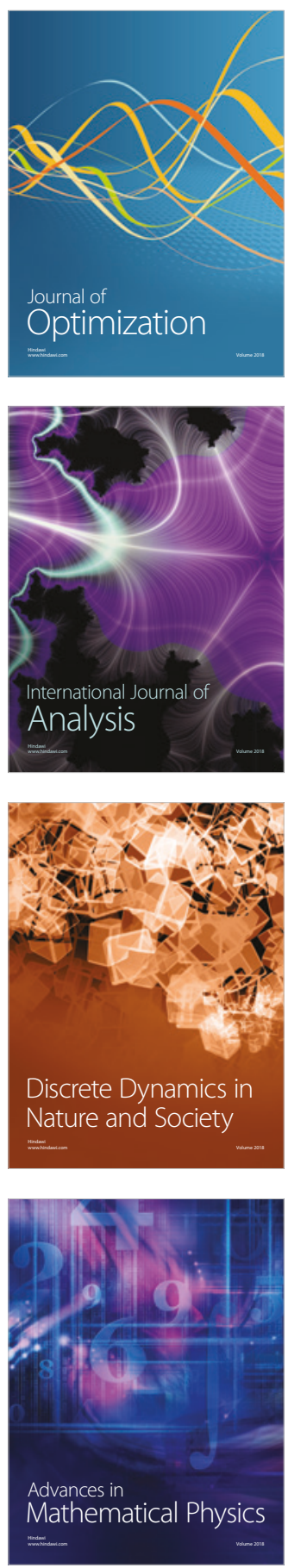